\title{
Application of FOS and CPP in intestinal health of Weaned Piglets
}

\author{
Feng Zhao ${ }^{1}$, Zhongsheng Xia ${ }^{2}$ \\ ${ }^{1}$ College of Agriculture and Forestry and Medical Science and Technology, Journal of Hainan Radio \& TV University, Haikou 570203, \\ China; \\ ${ }^{2}$ College of Animal Science and Technology, Guangxi University, Nanning 530004, China
}

\begin{abstract}
The present experiment was conducted to study the effects of different levels of oligosaccharide(FOS) and complex CPP preparation (CPP) in diets on growth performance and serum biochemical parameters for weaned piglets. 240 crossbred DLY( Duroc $\times$ Landrace $\times$ Yorkshire) 21-day-old weaning piglets, about $7.2 \mathrm{~kg}$ in initial body weight were selected and randomly divided into 6 groups, with 2 repeats for each group, 20 each repeat(half male and half female.). Group 1, as the control group,fed base $\operatorname{diet}(\mathrm{BD})$, group 2 fed BD adding $0.10 \%$ aureomycin, group 3, 4, 5, 6, respectively, fed BD adding $0.05 \%$ CPP, $0.05 \%$ FOS, $0.20 \%$ FOS and $0.10 \%$ FOS $+0.05 \%$ of CPP. Feeding trial was conducted for 35 days.Results: (1) Intestinal $\mathrm{pH}$ : Compared with the control group, the $\mathrm{pH}$ values of jejunum, ileum, cecum and colon in the 3, 4, 5 and 6 groups were significantly lower $(\mathrm{P}<0.05)$, while there was no significant difference between the antibiotic group and the control group $(\mathrm{P}>0.05)$. (2) Small intestinal villus morphology: Duodenal segment, villus length of 6 groups was significantly increased compared with the control group $(\mathrm{P}<0.05)$, villus height to recess depth ratio $(\mathrm{V} / \mathrm{C})$ was significantly increased $(\mathrm{P}<0.05)$. There was no significant difference in villus length between groups in jejunal segment $(\mathrm{P}>0.05)$. Groups five $\mathrm{V} / \mathrm{C}$ was significantly higher than that in the control group $(\mathrm{P}<0.05)$. Compared with the control group and the antibiotic group, the ileal segment in the six groups significantly increased $\mathrm{V} / \mathrm{C}(\mathrm{P}<0.05)$. (3) Intestinal flora: Adding FOS, CPP and their combination could reduce the number of E. coli and Salmonella in different intestinal segments, and increase the number of Lactobacillus and Bifidobacteria. Among them, the effect of joint addition is the most obvious. Conclusion: Adding FOS and C PP to the diet of weaned piglets could effectively reduce the intestinal $\mathrm{pH}$ value of piglets, increase the length of intestinal villi and $\mathrm{V} / \mathrm{C}$, reduce the number of harmful bacteria and increase the number of beneficial bacteria. It is beneficial to maintain the balance of intestinal morphology and microflora and improve the intestinal health of piglets. Among them, the combination of $0.10 \%$ FOS and $0.05 \%$ CPP is the best, followed by $0.20 \%$ FOS.
\end{abstract}

\section{Introduction}

Early weaning piglets are vulnerable to diarrhea, intestinal flora imbalance, decreased intake, slow growth, reduced immunity and other weaning stress syndrome. It has adverse effects on growth and intestinal health of piglets, Causing enormous economic losses. ${ }^{[1-2]}$ For a long time, feeding antibiotics has been used to prevent and treat diseases of weaned piglets, alleviate stress and promote growth. Therefore, research and development of antibiotic substitutes, maintenance or improvement of intestinal health of piglets through nutritional regulation technology, implementation of healthy breeding, and ensuring sustainable development of animal production have become one of the hotspots in animal nutrition research. ${ }^{[3]}$ In this experiment, FOS and compound CPP were added to the diet to study the effect of substituting antibiotics on intestinal health of piglets, so as to provide scientific basis for rational utilization of FOS and compound CPP in healthy pig breeding.

\section{Materials and methods}

\subsection{Test material}

FOS : FOS content $30 \%$; CPP : It mainly contains Clostridium butyricum, Bacillus subtilis, etc. Number of viable bacteria $3 \times 109 / \mathrm{g}$ 。Aureomycin premix,(AP) ,

Marketed products, aureomycin content $10 \%$ 。

\subsection{Laboratory Animals and Groups}

240 crossbred DLY (Duroc $\times$ Landrace $\times$ Yorkshire) 21-day-old weaning piglets, about $7.2 \mathrm{~kg}$ in initial body weight were selected and randomly divided into 6 groups, 
with 2 repeats for each group, 20 each repeat(half male and half female.). Group 1, as the control group,fed base $\operatorname{diet}(\mathrm{BD})$, group 2 fed BD adding $0.10 \%$ aureomycin, group 3, 4, 5, 6, respectively, fed BD adding $0.05 \%$ CPP, $0.05 \%$ FOS, $0.20 \%$ FOS and $0.10 \%$ FOS $+0.05 \%$ of CPP. Feeding trial was conducted for 35 days. Nutritional requirements of $8-20 \mathrm{~kg}$ piglets recommended by NY/T65-2004 for basic diets.

\subsection{Feeding management}

Before the experiment, the whole pigsty was completely disinfected and empty for more than 7 days. Transfer selected test pigs into sterilized pigsty, Weaning piglets around 21 days old after grouping, pre-test for 7 days, test period $35 \mathrm{~d}$. The pigsty was naturally illuminated, well ventilated and suitable for temperature and humidity. Feed 2-3 times a day. Feed freely and drink freely with a little surplus after each meal. Mmunization vaccination and feeding management were carried out according to the routine methods in pig farms. Pigsty are cleaned once a day and maintaining hygiene. Observation of pig feeding, fecal and urinary excretion and other behaviors at any time. The feed intake of piglets was recorded. The situation of diarrhea in pigs is Observed and recorded.

\section{Sample Collection}

\subsection{Measure Morphology of small intestinal mucosa}

At the end of feeding experiment, two healthy piglets were selected from each group (bloodletting slaughter). Place the piglets on the sterile operating table, the abdominal cavity was opened quickly with aseptic scalpel and scissors, and the intestinal segments were cut longitudinally. The duodenum (1.5 $\mathrm{cm}$ from pylorus), the middle jejunum (1.0 cm from pylorus), and the ileum $(2$ $\mathrm{cm}$ from the front of ileocecal valve) were cut. The intestinal contents were washed with saline and fixed in $4 \%$ paraformaldehyde fixative, and then observing of paraffin section, HE staining and tissue section. After dyeing, the prepared sections were observed and photographed under YS100 (nikon) optical microscope.

\section{2 $\mathrm{PH}$ value of chyme in different intestinal segments}

Combining with the measurement of small intestinal mucosa morphology, the intestine was carefully removed. And the Chyme of jejunum, ileum, cecum and colon segments were removed respectively. The $\mathrm{pH}$ values of jejunum, ileum, cecum and colon chyme were measured by $\mathrm{PHB}-4$ portable precise $\mathrm{pH}$ meter.

\subsection{Microbial flora determination of intestinal contents}

On the day of the end of the experiment, the jejunum, ileum, cecum and colon were ligated with cotton thread in combination with the $\mathrm{PH}$ value of chyme in each intestinal segment, and then quickly transfer to the aseptic operating table, mix evenly and take out the contents of each intestinal rupture. Take $0.5 \mathrm{~g}$ content from each sample, such as $4.5 \mathrm{~mL}$ sterile water into 10 $\mathrm{mL}$ centrifugal tube. Samples were made by oscillating on the oscillator for 1 minute. The intestinal contents were diluted reasonably by 10 -fold dilution method. The number of Escherichia coli, Salmonella, Lactobacillus and Bifidobacteria in jejunum, ileum, colon and cecum was determined by plate method.

\section{Result analysis}

\subsection{Effects of FOS and CPP on intestinal pH of Weaned Piglets}

The determination of $\mathrm{pH}$ value of each intestinal segment of piglets is shown in Table 1. Table 1 show, different levels of FOS and CPP had significant effects on intestinal $\mathrm{pH}$ of weaned piglets $(\mathrm{P}<0.05)$. The $\mathrm{pH}$ values of jejunum, cecum and colon were determined. 3, 4, 5 and 6 groups were significantly lower than control group (group 1) and antibiotic group (group 2). Ileum $\mathrm{pH}$ values in groups 3, 4 and 5 were significantly lower than those in groups 1 and $2(\mathrm{P}<0.05)$, while there was no significant difference in ileum, cecum and colon $\mathrm{pH}$ between groups 2 and group $1(\mathrm{P}>0.05)$.

Table 1. Determination of intestinal $\mathrm{pH}$ of piglets in each group

\begin{tabular}{ccccc}
\hline Group & Jejunal pH & Ileum pH & Cecum pH & Colon pH \\
\hline Group 1 & $6.34^{\mathrm{a}} \pm 0.10$ & $6.15^{\mathrm{a}} \pm 0.07$ & $5.72^{\mathrm{a}} \pm 0.07$ & $5.75^{\mathrm{a}} \pm 0.06$ \\
Group 2 & $6.08^{\mathrm{b}} \pm 0.08$ & $6.07^{\mathrm{a}} \pm 0.09$ & $5.73^{\mathrm{a}} \pm 0.06$ & $5.75^{\mathrm{a}} \pm 0.06$ \\
Group 3 & $5.82^{\mathrm{c}} \pm 0.14$ & $5.56^{\mathrm{c}} \pm 0.12$ & $5.46^{\mathrm{b}} \pm 0.06$ & $5.45^{\mathrm{c}} \pm 0.05$ \\
Group 4 & $5.93^{\mathrm{c}} \pm 0.09$ & $5.48^{\mathrm{c}} \pm 0.07$ & $5.41^{\mathrm{b}} \pm 0.03$ & $5.42^{\mathrm{c}} \pm 0.05$ \\
Group 5 & $5.79^{\mathrm{c}} \pm 0.10$ & $5.56^{\mathrm{c}} \pm 0.07$ & $5.47^{\mathrm{b}} \pm 0.05$ & $5.55^{\mathrm{b}} \pm 0.06$ \\
Group 6 & $5.83^{\mathrm{c}} \pm 0.08$ & $5.95^{\mathrm{b}} \pm 0.10$ & $5.27^{\mathrm{c}} \pm 0.08$ & $5.23^{\mathrm{d}} \pm 0.04$ \\
\hline
\end{tabular}

Note:The test data is expressed by means \pm SD. In the same column,values with different letter superscripts mean significant difference $(\mathrm{P}<0.05)$, while with the same or no letter superscripts mean no significant difference $(\mathrm{P}>0.05)$, The same as below. 


\subsection{Effects of FOS and CPP on villus morphology of small intestine in Weaned Piglets}

Table 2. Morphological determination of duodenal villi of piglets in each group

\begin{tabular}{ccccc}
\hline Group & Villus length $/ \mu \mathrm{m}$ & Villus width $/ \mu \mathrm{m}$ & crypt depth $/ \mu \mathrm{m}$ & Villus height/recess depth \\
\hline Group 1 & $299.77^{\mathrm{c}} \pm 0.22$ & $70.08^{\mathrm{c}} \pm 0.10$ & $108.75^{\mathrm{a}} \pm 0.12$ & $2.77^{\mathrm{c}} \pm 0.24$ \\
Group 2 & $334.53^{\mathrm{a}} \pm 0.38$ & $72.42^{\mathrm{bc}} \pm 0.38$ & $104.42^{\mathrm{ab}} \pm 0.17$ & $3.22^{\mathrm{b}} \pm 0.24$ \\
Group 3 & $315.68^{\mathrm{bc}} \pm 0.26$ & $74.75^{\mathrm{abc}} \pm 0.26$ & $107.92^{\mathrm{bc}} \pm 0.11$ & $2.94^{\mathrm{bc}} \pm 0.17$ \\
Group 4 & $309.54^{\mathrm{b}} \pm 0.22$ & $78.50^{\mathrm{ab}} \pm 0.22$ & $95.92^{\mathrm{a}} \pm 0.18$ & $3.23^{\mathrm{ab}} \pm 0.43$ \\
Group 5 & $318.34^{\mathrm{bc}} \pm 0.13$ & $79.17^{\mathrm{abc}} \pm 0.14$ & $93.50^{\mathrm{c}} \pm 0.19$ & $3.43^{\mathrm{ab}} \pm 0.29$ \\
Group 6 & $342.94^{\mathrm{a}} \pm 0.23$ & $83.08^{\mathrm{a}} \pm 0.23$ & $95.17^{\mathrm{bc}} \pm 0.16$ & $3.62^{\mathrm{a}} \pm 0.31$ \\
\hline
\end{tabular}

Note:The test data is expressed by means \pm SD. In the same column,values with different letter superscripts mean significant difference $(\mathrm{P}<0.05)$, while with the same or no letter superscripts mean no significant difference $(\mathrm{P}>0.05)$, The same as below .

The results of duodenal villus morphology of weaned piglets in each group are shown in Table 2. Table 2 show, There were significant differences in duodenal villus length, villus width, recess depth, villus height/recess depth ratio (V/C) between different levels of FOS and C PP $(\mathrm{P}<0.05)$.

The length of duodenal villus in group 6 (FOS + CPP) was significantly higher than that in group $1(14.40 \%$ (P $<0.05$ ), but there was no significant difference with that in antibiotic group $(\mathrm{P}>0.05)$. The villus width of 6 groups was significantly higher than that of 1 and 2 groups $(\mathrm{P}<0.05)$, and the recess depth of 5 groups was significantly lower than that of 1 and 2 groups $(\mathrm{P}<0.05)$. As for $\mathrm{V} / \mathrm{C}$, the ratio of 6 groups was 3.62, which was higher than $30.69 \%(\mathrm{P}<0.05)$ and $12.42 \%(\mathrm{P}>0.05)$ in group 1 and 2 , respectively.

\subsection{Effects of FOS and CPP on intestinal flora of Weaned Piglets}

Table 3. Determination of Jejunal Microflora in Piglets of Different Groups $\left(1 \mathrm{~g} \mathrm{cfu} \cdot \mathrm{g}^{-1}\right)$

\begin{tabular}{ccccc}
\hline Group & Escherichia coli & Salmonella & Lactobacillus & Bifidobacterium \\
\hline Group 1 & $7.54^{\mathrm{a}} \pm 0.13$ & $3.50^{\mathrm{a}} \pm 0.12$ & $7.49^{\mathrm{e}} \pm 0.03$ & $7.47^{\mathrm{c}} \pm 0.06$ \\
Group 2 & $7.30^{\mathrm{bc}_{0}} \pm 0.06$ & $3.21^{\mathrm{abc}} \pm 0.06$ & $7.76^{\mathrm{d}} \pm 0.10$ & $7.45^{\mathrm{c}} \pm 0.10$ \\
Group 3 & $7.50^{\mathrm{ab}} \pm 0.03$ & $3.30^{\mathrm{bc}} \pm 0.08$ & $8.39^{\mathrm{b}} \pm 0.09$ & $7.74^{\mathrm{b}} \pm 0.08$ \\
Group 4 & $7.23^{\mathrm{cd}} \pm 0.09$ & $3.34^{\mathrm{ab}} \pm 0.08$ & $8.59^{\mathrm{ab}} \pm 0.13$ & $8.20^{\mathrm{a}} \pm 0.08$ \\
Group 5 & $7.32^{\mathrm{bc}} \pm 0.05$ & $3.08^{\mathrm{c}} \pm 0.08$ & $8.09^{\mathrm{c}} \pm 0.09$ & $7.87^{\mathrm{b}} \pm 0.06^{\mathrm{b}}$ \\
Group 6 & $6.91^{\mathrm{e}} \pm 0.11$ & $3.13^{\mathrm{b}} \pm 0.08$ & $8.63^{\mathrm{a}} \pm 0.08$ & $8.21^{\mathrm{a}} \pm 0.08$ \\
\hline
\end{tabular}

Note:The test data is expressed by means \pm SD. In the same column,values with different letter superscripts mean significant difference $(\mathrm{P}<0.05)$, while with the same or no letter superscripts mean no significant difference $(\mathrm{P}>0.05)$, The same as below .

The results of jejunal flora of piglets in each group are shown in Table 3. Table 3 shows that the effects of different levels of FOS and CPP on jejunal flora of weaned piglets are significant $(\mathrm{P}<0.05)$. Escherichia coli jejuni in group 6 was the least, significantly lower than that in group 1 and group 2, with a decrease of 8.36\% $(\mathrm{P}<0.05)$ and 5.34\% $(\mathrm{P}<0.05)$, salmonella, 5 and 6 groups were significantly lower than that in group 1 ( $\mathrm{P}<0.05)$, lactobacillus, $3,4,5$ and 6 groups were significantly higher than that in group 1 and $2(\mathrm{P}<0.05)$, and Lactobacillus in group 6 was higher than that in group 1 and $2(\mathrm{P}<0.05)$, and bifidobacterium, 4, 5 and 6 groups were significantly higher than that in group 1 and 2.

\section{Discuss}

\subsection{Effects of FOS and CPP on intestinal pH of Weaned Piglets}

The results showed that the $\mathrm{pH}$ values of jejunum, ileum, cecum and colon of weaned piglets decreased with the addition of FOS and CPP. When $0.02 \%$ FOS and $0.10 \%$ FOS and 0.05 CPP were added together, the $\mathrm{pH}$ value of intestinal tract decreased most obviously. This is consistent with the experimental results of Xiao Hongde ${ }^{[5]}$ and Dou Maoxin ${ }^{[6]}$. This may be related to FOS and CPP acting alone or synergistically to promote the growth and reproduction of beneficial bacteria such as 
intestinal lactic acid bacteria and secrete large amounts of lactic acid.

\subsection{Effects of FOS and CPP on villus morphology of small intestine in Weaned Piglets}

By observing the villus morphology of duodenum, jejunum and ileum of weaned piglets, it was found that adding FOS and C PP alone or in combination could affect the villus length, villus width, recess depth, ratio of villus height to recess depth ( $\mathrm{V} / \mathrm{C}$ value). In the duodenal segment, the effect of $0.10 \%$ FOS and $0.05 \%$ CPP was better. The villus length and $\mathrm{V} / \mathrm{C}$ value were higher than those of the control group and $\%$ antibiotics group in varying degrees. In the jejunal segment, $0.20 \%$ FOS was effective, villus length and $\mathrm{V} / \mathrm{C}$ value were higher than those of control group and antibiotic group respectively. In ileal segment, the combination of $0.10 \%$ FOS and $0.05 \%$ CPP had better effect: significantly increased V/C value $(\mathrm{P}<0.05)$. Zhang Xiaoping. ${ }^{[4]}$ Adding CPP to the diet of weaned piglets, the effect of CPP group was similar to that of antibiotics group. This experiment is basically consistent with previous studies. Considering the villus morphology of the intestinal tract of weaned piglets, the effect of adding $0.10 \%$ FOS and $0.05 \%$ CPP is better. It can effectively increase the villus length and $\mathrm{V} / \mathrm{C}$ value, improve the shape and structure of the small intestine, and facilitate the digestion and absorption of nutrients in the diet.

\subsection{Effects of FOS and CPP on intestinal flora of Weaned Piglets}

Adding FOS and CPP to the diet of weaned piglets can effectively reduce the number of Escherichia coli and Salmonella, and effectively increase the number of Lactobacillus and Bifidobacteria. Adding 0.10\% FOS and $0.05 \%$ CPP to the diet could significantly reduce the number of E. coli and Salmonella in jejunum, ileum, cecum and colon segments, and increase the number of Lactobacillus and Bifidobacteria $(\mathrm{P}<0.05)$.

\section{Conclusion}

Adding FOS and CPP instead of antibiotics in diet could increase the ratio of villus length and villus height to crypt depth, improve intestinal morphology, promote intestinal repair and development, and enhance intestinal physical barrier and immune barrier. Increasing the number of beneficial bacteria such as Lactobacillus and Bifidobacterium, reducing the number of harmful bacteria such as Escherichia coli and Salmonella, maintaining the balance of intestinal microflora, and giving full play to the physiological role of dominant beneficial bacteria. Pathogen adhesion sites are competed. They could reduce intestinal $\mathrm{pH}$, secrete antimicrobial factors, maintain intestinal health, enhance intestinal immunity, increase disease resistance, promote digestion and absorption of nutrients and growth of piglets, improve breeding efficiency.
Considering the results of the experiment and the specific conditions of the pig farm, the combination of $0.10 \%$ FOS and $0.05 \%$ CPP in the diet was the best in piglet production practice, followed by $0.20 \%$ FOS.

\section{Reference}

1. HEO J M, OPAPEJU $F$ O, PLUSKE $J$ R, et al. Gastroin-testinal health and function in weaned pigs: a review of feeding strategies to control post-weaning diarrhoea without using in-feed antimicrobial compounds[J]. Journal of Animal Physiology and Animal Nutrition,2013, 97(2): 207 $-237$.

2. Dai Bing. Application of Nutritional Regulators for Intestinal Health of Weaned Piglets [D].Nanjin: Nanjing Agricultural University, 2011.

3. Yin Yu Long, Wu Xin, Tang Zhi Ru, Gao Yun Cen etc.Effects of L-Arginine Derivate on Growth Performance and Diarrhea of Weaned Piglets,2008, 29 (6) : 723-725,760.

4. Zhang Xiao Ping. Effects of functional oligosaccharides on intestinal mucosal morphology and dietary apparent digestibility of DM, OM, N, Ca and $\mathrm{P}$ in Weaned Piglets [D].Shanxi Taigu: Shanxi Agricultural University, 2003.

5. Xiao Hong De. An experimental study on the substitution of CPP for antibiotics in the diet of Weaned Piglets [D].Wuhan: Huazhong Agricultural University, 2013.

6. Dou Mao Xin, Wu Tao. Effects of Different CPP on Intestinal Microflora, $\mathrm{pH}$ and Volatile Base Nitrogen in Weaned Piglets [J]. Chinese Animal Husbandry and Veterinary Medicine, 2013, 40(2):84-87. 